\title{
Harmonisation globale des systèmes de brevet et impacts locaux
}

Brevet et accès aux médicaments dans les pays en développement sous AADPIC et AADPIC plus

\section{Samira Guennif}

\section{(2) OpenEdition Journals}

Édition électronique

URL : http://journals.openedition.org/ei/355

DOI : 10.4000/ei.355

ISSN : 2553-1891

Éditeur

Association Économie et Institutions

Édition imprimée

Date de publication : 30 octobre 2007

Pagination : 251-284

ISSN : $1775-2329$

Référence électronique

Samira Guennif, « Harmonisation globale des systèmes de brevet et impacts locaux », Économie et institutions [En ligne], 10-11 | 2007, mis en ligne le 31 janvier 2013, consulté le 30 avril 2019. URL : http://journals.openedition.org/ei/355 ; DOI : 10.4000/ei.355 


\title{
Harmonisation globale des systèmes de brevet et impacts locaux : Brevet et accès aux médicaments dans les pays en développement sous AADPIC et AADPIC plus*.
}

\author{
Samira GUENNIF ${ }^{1}$
}

\section{Introduction}

Selon les dernières estimations de l'ONUSIDA (2008), environ 33 millions de personnes vivraient avec le VIH/Sida dans le monde, pour la plupart dans les pays en développement (PED). L'Afrique Sub-Saharienne continuerait de payer le plus lourd tribut avec 22 millions d'adultes et d'enfants infectés. Par ailleurs, au regard d'une démographie importante, l'infection et les taux de prévalence progresseraient de façon significative en Asie. Actuellement, près de 5 millions de personnes vivraient avec la maladie dans la région, pour l'essentiel en Inde et en Chine.

$\mathrm{Si}$ un vaccin n'est toujours pas disponible, plus d'une vingtaine de traitements anti-rétroviraux (ARV) existent et permettent aux personnes de vivre avec l'infection. Des progrès importants ont été réalisés depuis les quelques monothérapies développées à la fin des années 80 jusqu'aux récentes multithérapies mises au point pour prévenir les risques de résistance chez les patients. A ce jour, ces derniers peuvent bénéficier de mono, bi et trithérapies, développer moins de résistances et bénéficier d'une amélioration sensible de qualité de vie au quotidien.

L'accès aux ARV est un enjeu majeur dans les PED où l'épidémie a des effets socio-économiques dramatiques comme le notent les études micro et macro-économiques réalisées sur l'impact $\mathrm{du} \mathrm{VIH} /$ Sida sur les ménages ou la croissance économique (Over, 1992, Ainsworth, 1993, Bonnel, 2000, Dixon et al., 2001, Drouin et al., 2003). Dans l'ensemble, cette épidémie rappelle que l'accès aux traitements n'est pas un enjeu nouveau dans les PED. Depuis les années 70 , l'Organisation mondiale de la santé (OMS) travaille pour le "droit de tous à la santé " en cherchant principalement à augmenter l'accès aux "médicaments essentiels" dans les pays

\footnotetext{
* Cet article a été réalisé avec le soutien financier de l'Agence Nationale de Recherches sur le Sida, de Sidaction et le Centre de Sciences Humaines de Delhi.

${ }^{1}$ CEPN, UMR-CNRS 7115, Université Paris 13.
} 
pauvres. Dans cette perspective, elle a édité en 1977 la première liste de médicaments essentiels. Celle-ci désigne les médicaments les moins chers qui permettent de traiter les infections les plus prégnantes dans les PED. Depuis, le médicament a été défini comme un bien spécifique essentiel qui doit être rendu accessible au plus grand nombre à un prix raisonnable. En particulier, pour assurer l'accès des patients aux ARV dans les PED, l'OMS et l'ONUSIDA ont lancé en 2003 l'initiative " 3 by 5 ". L'objectif est de fournir des ARV à trois millions de personnes infectées dans les pays à revenu bas ou moyen d'ici la fin de l'année 2005. Il s'agit d'une étape essentielle vers un objectif plus large, celui d'assurer un accès universel aux traitements anti-sida pour tous ceux qui en ont besoin dans les pays pauvres.

Pour cela, l'OMS (2006) publie et révise régulièrement des recommandations pour une approche de santé publique concernant la fourniture d'ARV dans les pays à ressources limitées. Concrètement, ces recommandations indiquent aux autorités sanitaires quand commencer un traitement antisida, quel traitement de première intention utiliser pour les adolescents, les adultes ou les femmes enceintes, en considération essentiellement de l'efficacité de ces traitements, de leurs effets secondaires et de leurs prix ? Les trithérapies sont fortement conseillées dans la mesure où les risques de résistance sont grandement réduits.

Or, l'accès aux traitements dans les PED frappés par des épidémies telles celle du VIH/Sida, pose également la question du statut juridique de ces médicaments et les évolutions récentes enregistrées dans le domaine des droits de propriété intellectuelle (DPI) jouent un rôle considérable. C'est pourquoi, cet article vise à examiner l'influence de ces évolutions juridiques sur l'accès aux médicaments dans les PED. Précisément, focalisant sur les vecteurs essentiels de l'évolution des DPI dans le monde, l'Accord sur les Aspects de Droits de Propriété Intellectuelle touchant au Commerce (AADPIC) et les Accords de Libre Echange (ALE) états-uniens, il est montré que ces accords internationaux donnent lieu à des systèmes de DPI globaux contraignants qui peuvent avoir des effets locaux préjudiciables pour la santé publique au Sud. Notre argument est que de nombreuses dispositions manquantes, ouvertes ou excessivement contraignantes dans ces accords permettent d'étendre les monopoles détenus par les entreprises, de prévenir la concurrence générique et de limiter l'accessibilité des traitements dans les PED. Plus encore, certains faits stylisés tirés des expériences sanito-industrielles de l'Inde et de la Thaïlande révèlent que ces craintes pour la santé publique ne sont pas simplement hypothétiques mais déjà palpables, particulièrement dans le cas de l'accès des populations aux traitements antisida. 
Pour ce faire, les objectifs et principes de l'AADIPC seront tout d'abord présentés (§2). Au-delà des obligations imposées pour la protection effective de la propriété intellectuelle, des considérations sanitaires sont introduites. Des exceptions au brevet ou des flexibilités existent pour promouvoir la santé publique et l'accès aux traitements dans des circonstances particulières. Puis, nous examinerons les ALE signés et négociés dans les PED par les EtatsUnis après la ratification de l'AADPIC (§3). Ces accords imposent une protection des DPI qui va bien au-delà des standards imposés par l'Organisation mondiale du commerce $(\mathrm{OMC})$, d'où leur appellation d'" AADPIC plus ". Les aspects de santé publique y sont négligés et notamment le recours aux flexibilités prévues par l'AADPIC y est limité. Enfin, l'analyse de faits saillants nous permettra de questionner la compatibilité entre régimes de DPI contraignants et accès aux traitements au Sud (§4). D'une part, comme en témoigne la trajectoire indienne, il semble qu'à ce jour le moyen le plus efficace de favoriser l'accès des populations aux traitements soit la mise en place d'un régime de DPI souple. Ce faisant, l'Inde entend depuis 2005 tout mettre en oeuvre pour poursuivre ses efforts sanitaires. Actuellement, elle amende sa loi sur le brevet pour la rendre compatible avec l'AADPIC en tirant le meilleur parti des flexibilités prévues. D'autre part, analysant la trajectoire thaïlandaise, nous constaterons que le renforcement d'un système de DPI peut nuire à l'accessibilité des traitements comme le suggèrent les difficultés rencontrées en pratique par la Thaïlande pour assurer l'accès des patients infectés par le VIH/Sida aux thérapies à travers l'usage de flexibilités.

\section{Le renforcement des régimes de DPI : de l'AADPIC à la déclaration ministérielle de Doha}

Depuis des décennies, les régimes de DPI connaissent des renforcements importants dans le monde. Ratifié en 1994, l'AADPIC est progressivement mis en place dans les PED Membres de l'OMC. Dans le secteur pharmaceutique, cet accord institue un régime de DPI fort en reconnaissant les brevets à la fois sur les procédés de fabrication et les produits, en allongeant la durée du brevet à vingt ans minimum et en étendant sa couverture géographique à l'ensemble des pays Membres de l'OMC (Nogues, 1990, Desterbecq et Remiche, 1996, Abbott, 1996, Boulet et Velasquez, 1999, Correa, 2000). Ainsi, les législations nationales doivent être amendées et mises en conformité avec l'AADPIC.

\subsection{Objectifs et principes de l'AADPIC}


$\mathrm{Au}$ début des années 80, arguant du fait que les délais réglementaires réduisaient en pratique la durée de vie des brevets, l'industrie pharmaceutique états-unienne a commencé à plaider pour une extension de la durée du brevet. En 1984, les autorités du pays accèdent à leur requête en portant la durée du brevet à 20 ans après dépôt ${ }^{2}$. L'Europe suivra quelques années plus tard, suivant en cela l'exemple français dont le Certificat complémentaire de protection étend la durée du brevet de 5 ans minimum. En 1992, l'Europe adopte donc le Certificat communautaire qui prolonge le brevet de 5 ans minimum (Mfuka, 2002).

En outre, de nombreuses études insistent sur les préjudices que subirait l'industrie pharmaceutique. L'attention est portée sur le coût croissant des programmes de recherche et développement $(R \& D)$. Des millions de dollars seraient engloutis dans le développement d'un médicament, depuis les phases de recherche sur ses qualités thérapeutiques jusqu'à son lancement commercial (Grabowsky, 1982, DiMasi et al., 1991, Goozner, 2005). Parallèlement, les PED sont sous la pression des pays développés (PD). Ainsi, 1'Inde est accusée de piraterie, comportement qui porterait atteinte aux profits des multinationales, mettrait en danger le développement de nouveaux médicaments et menacerait le bienêtre global ${ }^{3}$. Les PD prennent position lors des forums gouvernant le commerce international et cherchent à imposer des régimes de DPI plus contraignants au Sud. Néanmoins, l'Inde et le Brésil font campagne pour éviter que des négociations sur les DPI ne soient ouvertes pendant le cycle de l'Uruguay.

Ce cycle a duré huit ans et s'est achevé en avril 1994 avec la création de l'OMC et la ratification de l'AADPIC à Marrakech. Tout pays Membre doit se soumettre à ses règles et observer le contenu de cet accord. A défaut, les contrevenants s'exposent à des sanctions commerciales.

L'OMC établit tout d'abord que l'objectif de l'AADPIC est de mettre en œuvre des standards internationaux minimums concernant la protection de la propriété intellectuelle (Velasquez et Boulet, 1999, Raizada et Sayed, 2002). Autrement dit, l'accord n'institue pas un régime de DPI unique et universel. Les membres doivent observer ces standards minimums par les voies et les moyens qu'ils auront choisis. Ils sont libres également d'adopter un régime plus contraignant que celui prévu dans l'AADPIC (Article 1).

Cet objectif ne constitue pas une "obligation absolue et unique" (Velasquez et Boulet, 1999). L'OMC reconnaît la nécessité pour les Membres de satisfaire des objectifs en matière de

${ }^{2}$ Ou 14 ans après obtention d'une autorisation de mise sur le marché.

3 Accusation fallacieuse puisque l'Inde dispose alors d'un système de DPI plus souple où seuls les procédés de fabrication sont brevetables. Aussi, l'industrie domestique peut en toute légalité copier les molécules mises au point au Nord. 
développement et de santé publique. Ainsi, la protection par le brevet doit "contribuer à la promotion de l'innovation technologique et au transfert et à la diffusion de la technologie, à l'avantage mutuel de ceux qui génèrent et de ceux qui utilisent des connaissances techniques et d'une manière propice au bien-être social et économique, et à assurer un équilibre de droits et d'obligations " (Article 7).

Il suit que les Membres peuvent légiférer suivant des principes tels que "la santé publique, (...), et l'intérêt public dans des secteurs d'une importance vitale pour leur développement socio-économique et technologique " (Article 8.1). De même, ils peuvent mettre en œuvre "des mesures appropriées " pour "éviter l'usage abusif des droits de propriété intellectuelle par les détenteurs de droits ou le recours à des pratiques qui restreignent de manière déraisonnable le commerce ou sont préjudiciables au transfert international de technologie " (Article 8.2). En résumé, l'AADPIC n'a pas pour objet d'imposer une protection inconditionnelle des DPI.

L'article 27 circonscrit le champ de la brevetabilité. Il établit qu'" un brevet pourra être obtenu pour toute invention, de produit ou de procédé, dans tous les domaines technologiques ". Désormais, il n'est plus possible pour un pays d'exclure des produits et des secteurs industriels particuliers du champ du brevet. Plus encore, les critères de brevetabilité sont fixés: produits et procédés doivent être "nouveaux", impliqués "une activité inventive" et "susceptible

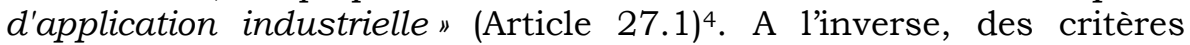
d'exclusion du champ du brevet sont précisés notamment "pour protéger l'ordre public ou la moralité " ou "pour éviter de graves atteintes à l'environnement" (Article 27.2). Enfin, "les méthodes diagnostiques, thérapeutiques et chirurgicales pour le traitement des personnes ou des animaux" ne sont pas brevetables (Article 27.3).

De plus, le brevet pour les procédés et les produits sera valable pour au moins vingt ans, à partir de la date du dépôt (Article 33). Précisément, seuls les médicaments ayant fait l'objet d'un dépôt de brevet après 1995 peuvent être brevetés dans les Etats Membres de l'OMC. En cas de différend et de procédure judiciaire, le détenteur n'aura pas à prouver la validité de son brevet; il incombera au " copieur " d'apporter la preuve de son invalidité (Article 34).

L'article 27 interdit aux Membres de recourir à des pratiques discriminantes lors de la délivrance et de l'usage d'un brevet. En respect du principe de traitement national (Article 3), l'usage d'un brevet doit être possible que les produits soient "importés ou, (...), d'origine nationale ". La notion de "working patent", ou l'usage effectif d'un brevet sur un territoire national sous la forme d'une production

\footnotetext{
${ }^{4}$ Etant entendu que "les expressions "activité inventive " et "susceptible d'application industrielle " pourront être considérées par un Membre comme synonymes, respectivement, des termes "non évidente " et " utile " ".
} 
locale, largement adoptée dans les PED est donc rendue impraticable.

Dans la mesure où l'AADPIC vise à instaurer un régime de DPI cohérent avec des objectifs de santé publique et de diffusion de l'innovation dans les PED, il est prévu des exceptions aux droits conférés par les brevets, des flexibilités. Les brevets peuvent être contournés dans des circonstances particulières.

\subsection{Les flexibilités prévues par l'AADPIC}

Tout d'abord, alors que l'AADPIC est entré en vigueur en 1995 dans les PD, une période de transition a été accordée aux PED (et les pays en transition) (Article 65). Puis, la fin de cette période transitoire a été fixée à 2005 pour les PED tels que la Chine et l'Inde, et à 2015 pour les pays les moins avancés. Toutefois, cette période pourra être prolongée pour ces derniers compte tenu de leurs "besoins et impératifs spéciaux" et leur "besoin de flexibilité pour se doter d'une base technologique viable " (Article 66.1). Ainsi, les pays africains par exemple devront se mettre en conformité avec l'AADPIC au plus tard en 2015 si la période de transition n'est pas prolongée 5 .

Dans le respect des principes de l'AADPIC, un pays peut contourner le brevet pour promouvoir des objectifs de santé publique comme l'accès aux médicaments. "Dans des situations d'urgence nationale ou d'autres circonstances d'extrême urgence", "en cas d'utilisation publique à des fins non commerciales " (Article $31 b)^{6}$ ou de pratique avérée anti-concurrentielle (Article 31c), un pays peut utiliser les droits conférés par un brevet sans l'autorisation de son détenteur ${ }^{7}$. Celui-ci doit simplement être informé dans un délai raisonnable et être compensé de façon adéquate. Dans le cas entre autres d'une épidémie de VIH/Sida, de paludisme ou de tuberculose, ou étant donné les prix prohibitifs pratiqués/les quantités commercialisées insuffisantes, un pays pourra donc délivrer une licence obligatoire (LO). Une agence publique ou une entreprise privée sera alors autorisée à produire un médicament pour qu'une urgence nationale soit traitée : des médicaments génériques plus abordables seront rendus disponibles. L'accord prévoit que la définition d'une urgence nationale reste à la discrétion des Etats.

Selon l'AADPIC, le détenteur d'un brevet a le droit de fabriquer, d'utiliser, de proposer à la vente, de vendre ou d'importer son produit (Article 28.1a). Il a également le droit de céder ses droits grâce à des contrats de licence (Article 28b). Le droit d'importer

\footnotetext{
${ }^{5}$ Si ces pays n'avaient pas, sous l'effet d'un autre accord international, reconnu par ailleurs les brevets sur les procédés et produits.

${ }^{6}$ Equivalent de la "Bolar provision " des Etats-Unis.

${ }^{7}$ Il n'est pas indispensable de chercher à obtenir une licence volontaire de la part du détenteur du brevet, c'est-à-dire le transfert volontaire des droits contre royalties.
} 
dépend du principe d'exhaustivité des droits retenu, principe selon lequel un détenteur peut conserver ou perdre certains droits. Ce principe couvre trois possibilités et conditionne la possibilité pour un pays de procéder à des importations parallèles (IP) :

Dans le cas d'un principe d'exhaustivité national, la circulation du produit est limitée au pays. Si le détenteur du brevet accepte que son produit soit commercialisé dans un pays, ce principe interdit que le produit soit réexporté vers un quelconque pays tiers.

Dans le cas d'un principe d'exhaustivité régional, la circulation du produit est circonscrite à une région. Si le détenteur $\mathrm{du}$ brevet accepte que son produit soit commercialisé dans l'Union Européenne par exemple, le principe régional prévoit que le produit pourra être réexporté dans un pays de l'Union en toute légalité. Toute réexportation vers un pays se situant hors de l'Union sera au contraire interdite.

- Enfin, dans le cas d'un principe d'exhaustivité international, la circulation du produit n'est nullement limitée. Une fois que le détenteur du produit a accepté que son produit soit commercialisé dans ce pays, ce principe autorise sa réexportation partout ailleurs.

Dans ce dernier cas de figure, un pays A pourra s'approvisionner en médicaments dans un pays B s'ils y sont vendus à un prix plus faible. Précisément, le principe d'exhaustivité adéquat doit être retenu dans les deux pays pour permettre au pays A de procéder à une IP et au pays $\mathrm{B}$ de procéder à une exportation parallèle. Dans le cas d'un principe d'exhaustivité régional, les pays $\mathrm{A}$ et $\mathrm{B}$ doivent naturellement appartenir à la même région : l'Union européenne, l'Organisation africaine de la propriété intellectuelle (OAPI), l'Organisation régionale africaine de la propriété intellectuelle (ARIPO), l'Accord de libre échange nord américain (NAFTA), l'association des nations de l'Asie du sud-est (ASEAN), etc. Du reste, l'IP est une mesure réglementaire qui permet de lutter contre les pratiques anti-concurrentielles et discriminantes (pratiques largement condamnées par l'OMC) et de rétablir le jeu de la concurrence, particulièrement lorsque les prix sont avérés prohibitifs ou les quantités disponibles jugées insuffisantes.

L'AADPIC établit qu'" aucune disposition du présent accord ne sera utilisée pour traiter la question de l'épuisement des droits de propriété intellectuelle" (Article 6). Les Membres ont toute latitude pour définir le principe retenu et les moyens prévus pour lutter contre des pratiques anti-concurrentielles, pour faciliter l'accès à des traitements plus abordables par voie d'IP. Cependant les débats sont vifs et les recommandations fusent en la matière. Certains rappellent qu'un principe d'exhaustivité international peut inciter les 
entreprises à opter pour une stratégie de prix unique dans le monde pour mettre un terme aux IP. En effet, les entreprises peuvent avoir pour souci de prévenir les effets indésirables des exportations parallèles depuis des pays où les médicaments sont commercialisés à des prix plus faibles vers ceux où les prix pratiqués sont plus élevés. Rationnellement, la mise en œuvre d'une stratégie de prix unique signifierait une révision considérable des prix à la hausse dans les PED et entamerait sérieusement l'accessibilité des traitements. Ce faisant, il est recommandé aux Membres de légiférer en faveur d'un principe d'exhaustivité national. A contrario, d'autres opposent à cette idée le fait que renoncer aux IP, c'est renoncer à la possibilité d'introduire plus de concurrence sur un marché et de tirer les prix vers le bas.

En résumé, l'AADPIC stipule un certain nombre de choses. Par exemple, les brevets sont accordés pour les procédés et les produits pour au moins 20 ans. Toutefois, certains points restent flous. Des dispositions sont ouvertes, laissées à la discrétion des Membres comme la définition d'une urgence nationale ou le principe d'exhaustivité des droits à adopter.

\subsection{La déclaration ministérielle de Doha : la santé publique doit prévaloir sur les DPI}

En considération des difficultés et des pressions subies par les PED pour mettre en pratique les flexibilités prévues par l'AADPIC, dues en grande partie aux imprécisions et ambiguités contenues dans certaines dispositions, les événements ont conduit les Membres à réaffirmer à Doha leur attachement au principe de protection des DPI, en tant que force motrice sous-tendant l'innovation, reconnaissant que: "la protection de la propriété intellectuelle est importante pour le développement de nouveaux médicaments " (paragraphe 3 de la déclaration ministérielle de Doha).

Puis, le principe selon lequel la protection des DPI est subordonnée à la santé publique fut réitéré : "nous convenons que l'Accord sur les ADPIC n'empêche pas et ne devrait pas empêcher les Membres de prendre des mesures pour protéger la santé publique". Par conséquent, "nous affirmons que ledit accord peut et devrait être interprété et mis en œuvre d'une manière qui appuie le droit des Membres de l'OMC de protéger la santé publique et, en particulier, de promouvoir l'accès de tous aux médicaments " (paragraphe 4 de la déclaration ministérielle de Doha). Fut donc réaffirmée la possibilité pour les Membres de recourir aux IP et LO dans le cas notamment d'une urgence nationale et de définir en toute discrétion ce qui constitue une urgence nationale (paragraphes $5 b$ et $5 c$ de la déclaration ministérielle de Doha). De surcroît, les Membres sont libres d'adopter leur principe d'exhaustivité des droits et d'établir 
ainsi l'étendue du recours possible aux IP (paragraphe 5d). Toutefois, pour répondre aux objections émises par les pays africains, dans l'impossibilité d'accorder des LO faute de capacités de production domestiques satisfaisantes, les Membres ont été chargés de trouver une solution d’ici 2003 (paragraphe 6 de la déclaration ministérielle de Doha).

En août 2003, quelques mois avant le sommet de Cancun, un accord a été trouvé. Une flexibilité additionnelle a été introduite : les Membres ont la possibilité de procéder à des importations sous LO. Ainsi, la Tanzanie peut accorder une LO et demander à une entreprise installée dans un pays tiers de produire et d'exporter vers son territoire des médicaments pour lui permettre notamment de traiter une urgence sanitaire. Des conditions ont été établies : entre autres volume déterminé de production, identification sans équivoque des produits, notification du pays de destination et rémunération adéquate du détenteur du brevet conformément à l'article $31 \mathrm{~h}$ de l'AADPIC.

Pour autant, la question de la diffusion de l'innovation dans le monde et spécialement l'enjeu des transferts technologiques vers les PED reste ouverte. Certes, la mise en oeuvre d'un cadre minimum global de protection des DPI peut permettre aux entreprises de recouvrer les ressources investies dans la $R \& D$ sous la forme d'un monopole industriel et commercial temporaire (Arrow, 1962, Demsetz, 1967, Grabowsky, 1982, Mansfield, 1986, Levin et al., 1987, Scherer, 1998, Cohen et al., 2000, Crampes, 2000). Certes, une régime de DPI contraignant peut alors inciter l'innovation et améliorer le bien être des populations grâce à la fourniture de nouveaux traitements (Cutler et MacClellan, 2001, Grabowski, 2002, Lichtenberg, 2002, NIHCM, 2002). Mais ce régime peut-il favoriser les transferts technologiques et fournir les PED en nouvelles innovations technique et thérapeutique (Mansfield, 1994, Saggi, 2000, Correa, 2001, Lall, 2003, Maskus, 2004, Correa, 2005, Maskus et Reichman, 2005, Gallagher, 2005) ?

De fait, en comparaison des outils déployés pour assurer la protection des DPI, force est de constater que les intentions gouvernent à ce jour concernant la promotion des transferts technologiques car des dispositions manquent concrètement. L'article 66.2 de l'AADPIC note que "les pays développés Membres offriront des incitations aux entreprises et institutions sur leur territoire afin de promouvoir et d'encourager le transfert de technologie vers les pays les moins avancés Membres pour leur permettre de se doter d'une base technologique solide et viable". Par ailleurs, le paragraphe 37 de la déclaration de Doha convient "d'un examen, au sein d'un Groupe de travail sous les auspices du Conseil général, de la relation entre commerce et transfert de technologie, et de toutes recommandations éventuelles sur les mesures qui pourraient être 
prises dans le cadre du mandat de l'OMC pour accroître les apports de technologie aux pays en développement ". Or, avec l'interdiction faite aux Membres de mettre en $œ u v r e$ ou de conserver la notion de " working patent ", il semble que le seul outil, qui aurait pu aider à la diffusion de l'innovation, aux transferts technologiques vers le Sud, ait disparu.

Mais tandis que des débats animés accompagnent la mise en place progressive de l'AADPIC dans les PED et qu'un intérêt certain s'exprime invitant les pays à amender leur loi sur le brevet de façon à tirer le meilleur parti des flexibilités prévues par l'accord, une tendance majeure autre se dessine. L'attention doit désormais et aussi se porter sur la trajectoire suivie par les Etats-Unis qui, loin du multilatéralisme défendu par l'OMC, négocient depuis cinq ans des ALE avec les PED. Le contenu de ces accords pourrait grandement remettre en cause la capacité des partenaires à recourir aux flexibilités prévues par l'AADPIC et menacer sérieusement la promotion de la santé publique dans les PED.

\section{Accords de libre échange états-uniens: 1'accès aux traitements sous des standards plus élevés}

Alors que des discussions vives portent sur l'impact préjudiciable de l'AADPIC sur le développement socio-économique des PED et l'accès des populations aux médicaments, les ALE se multiplient entre les Etats-Unis et ces pays. Leur contenu a déjà conduit les commentateurs à les qualifier d'" AADPIC plus " dans la mesure où le régime de protection des DPI institué est plus contraignant que celui prévu par l'AADPIC. Ce qui n'a pas été obtenu dans l'enceinte de l'OMC semble sur le point d'être largement obtenue dans le cadre plus feutré des accords bilatéraux et régionaux états-uniens.

\subsection{Les accords états-uniens de libre échange bilatéraux et régionaux}

Depuis cinq ans, des ALE ont été signés et d'autres sont en cours de négociation entre les PED et les Etats-Unis. Il s'agit d'accords bilatéraux ou régionaux. Un accord a été signé avec la Jordanie en 2000. Deux autres accords ont été établis en 2003 avec Singapour et le Chili. Ensuite, l'Australie, le Bahreïn et le Maroc ont passé un accord en 2004. Au cours de l'année 2006, des accords ont été signés avec Oman, la Colombie et le Pérou. D'autres accords bilatéraux sont en cours de négociation avec le Panama, la Thaïlande, les Emirats Arabes Unis, la Malaisie ou encore la Corée du Sud comme le rappellent les manifestations qui sont organisées 
ici et là par la Société civile ou les commentaires des autorités publiques sur le contenu et les effets de ces accords.

De même, les Etats-Unis passent des accords régionaux. Depuis l'ALE signé avec le Mexique et le Canada en 1993 (l'ALENA), d'autre accords régionaux ont suivi. Spécialement, l'ALE avec l'Amérique centrale a été signé en mai 2004. Cet accord implique le Costa Rica, le Salvador, le Guatemala, le Honduras, le Nicaragua. En parallèle, les Etats-Unis ont négocié séparément un accord bilatéral avec la République dominicaine, qui a rejoint quelques mois plus tard l'ALE avec l'Amérique Centrale.

D'autres accords régionaux sont en discussion. Depuis 2003, un ALE avec l'Union douanière Sud-africaine, composée de l'Afrique du Sud, du Botswana, de la Namibie, du Lesotho et du Swaziland, est en négociation ${ }^{8}$. Par ailleurs, des négociations ont commencé depuis 2004 pour arrêter un accord régional avec les pays andins : la Colombie, le Pérou, l'Equateur et la Bolivie. A ce jour, cinq cycles de négociations ont eu lieu avec trois de ces pays : la Colombie, le Pérou et l'Equateur. Des négociations sont également en cours pour parvenir à un accord avec les Emirats Arabes Unis. Elles serviront de tremplin pour un accord régional, pour la zone de libre échange du Moyen-Orient qui aura pour but de faire avancer les réformes économiques et de promouvoir le commerce entre les pays au MoyenOrient et dans le Golfe persique comme l'assurent invariablement les représentants du commerce des Etats-Unis. Pour l'instant, les liens économiques se multiplient et s'intensifient sur la base d'accordscadres de commerce et d'investissement, de traités bilatéraux d'investissement et des ALE passés avec notamment l'Egypte, le Koweït, le Qatar, l'Arabie Saoudite, la Tunisie ou encore le Yémen.

En résumé, les Etats-Unis mènent simultanément des négociations bilatérales et régionales avec des PED et ont pour objectif de mettre en œuvre des ALE sur une base régionale la plus large possible. Ainsi, les accords bilatéraux et régionaux étroits signés ou actuellement négociés avec les pays d'Amérique Latine devraient déboucher à terme sur la mise en place d'une zone de libre échange plus large, la zone de libre échange des Amériques. De même, pour faciliter un accord régional avec les Nations de l'Asie du Sud-est, les Etats-Unis multiplient les accords bilatéraux signés ou en négociation avec les Membres de cette association. Les dix Membres (le Brunei, le Cambodge, l'Indonésie, le Laos, la Malaisie, le Myanmar, les Philippines, Singapour, la Thaïlande et le Vietnam) représentent collectivement le cinquième partenaire commercial des Etats-Unis.

8 Créée en 1969, cette association a pour objectif de promouvoir le développement économique dans la zone sud-africaine. Depuis 1994, le commerce avec les Etats-Unis a cru de $300 \%$. Les exportations vers les Etats-Unis s'élèvent à 1,7 milliards de dollars contre 2,5 milliards pour les importations en 2002 . 
Pour dire les choses autrement, chaque fois que les négociations au sein d'un accord régional deviennent difficiles, des liens bilatéraux avec un ou plusieurs pays de la zone sont activés pour parvenir à des accords bilatéraux qui s'inséreront ensuite dans un arrangement plus vaste. Somme toute, de plus en plus de PED sont en train de signer des ALE avec les Etats-Unis sur une base bilatérale et/ou régionale ; la liste donnée ici n'étant pas exhaustive.

\subsection{Objectifs et propos des accords de libre échange états- uniens}

Chaque ALE contient un chapitre sur les DPI ${ }^{9}$. Celui-ci n'établit pas clairement les objectifs et les intentions du dit accord. Précisément, l'AADPIC et la déclaration ministérielle de Doha mettent en avant l'équilibre approprié à trouver entre la protection des DPI, la promotion de l'innovation et la diffusion des technologies, d'un côté, et la promotion du bien être économique et social, de l'autre (Musungu et Oh, 2006). Par contraste, les ALE n'explicitent pas de tels objectifs et tout naturellement des dispositions sont considérées comme des mesures pouvant limiter le recours aux flexibilités prévues par l'AADPIC. Or, l'utilisation de ces flexibilités peut faciliter la fourniture de médicaments génériques et permettre l'accès des patients à des médicaments plus abordables.

Sur ce point, les PED semblent considérer qu'il s'agit d'un jeu donnant-donnant où ils obtiendront un gain net en échange de concessions sur les DPI. En effet, un accès plus large aux marchés des PD, via des réductions de taxes d'importation, pourra procurer des revenus plus importants grâce aux exportations de produits agricoles (et autres produits). Le PIB et le revenu par tête s'en trouveront accrus dans ces pays. Finalement, les populations pourront se permettre d'acheter des médicaments plus chers ${ }^{10}$.

Dans quelques ALE bilatéraux ou régionaux (ALE Amérique centrale/République dominicaine/Etats-Unis, ALE Chili/Etats-Unis par exemple), les premières dispositions sont établies et les objectifs sont alors définis. "Les objectifs de cet Accord, tels qu'élaborés plus spécifiquement à travers ses principes et règles, y compris le traitement national, le traitement de la Nation la plus favorisée et la transparence, sont de:

\footnotetext{
${ }^{9}$ Le point de départ de ces accords est le "Special 301 report " qui recense l'ensemble des pays dont la législation, la politique ou les pratiques sont jugées préjudiciables aux intérêts des Etats-Unis. Sur la base du "Priority watch list ", ces pays sont sous la pression commerciale des Etats-Unis. Ce mécanisme a finalement conduit à la conclusion d'ALE entre les PED et les Etats-Unis durant les années 2000.

10 Toutefois, la question des subventions est ignorée dans ce débat. Les Etats-Unis continuent entre autres de subventionner la production domestique de coton, ce qui donne lieu à des différends au sein de l'OMC. On peut craindre ainsi que les bénéfices attendus dans l'agriculture soient surestimés.
} 
a) lever les barrières au commerce, et de faciliter les flux croisés de biens et services entre les territoires des deux Parties ;

b) promouvoir les conditions d'une concurrence équitable dans la zone de libre échange ;

c) accroître substantiellement les opportunités d'investissement sur le territoire des Partie;

d) fournir une protection adéquate et effective des droits de propriété intellectuelle sur le territoire de chaque Partie;

e) créer des procédures effectives pour la mise en place et l'application de cet Accord, pour une administration conjointe et pour la résolution des différends; et

f) établir un cadre pour de plus amples coopérations trilatérale, régionale et multilatérale pour étendre et augmenter les bénéfices de cet Accord,

En somme, il manque toute référence à une protection des DPI d'une manière consistante avec le bien-être économique et social des populations au moyen de la promotion de la santé publique. Les ALE focalisent sur la promotion du commerce et insistent essentiellement sur le besoin d'éliminer les obstacles au commerce et d'établir une protection effective des DPI. Ce point repose sur un postulat adopté par les Etats-Unis, implicitement ou explicitement comme dans le cas de l'ALE Chili/Etats-Unis: "la protection et la mise en exécution des droits de propriété intellectuelle constituent un principe fondamental de ce chapitre qui aide à promouvoir l'innovation technique et également le transfert et la diffusion des technologies pour le bénéfice mutuel des producteurs et des utilisateurs de technologies, et qui encourage le développement du bien être social et économique "(Chapitre 17, préambule). Tandis que les chercheurs s'interrogent encore sur les effets de la protection intellectuelle sur le développement industriel et le bien-être économique au Sud (Maskus et Reichman, 2005, Gallagher, 2005) ${ }^{11}$; pendant que 1'OMS diligente la Commission sur la propriété intellectuelle, l'innovation et la santé publique créée en 2003 de produire des données et des propositions pour une analyse des effets des DPI sur l'innovation et la santé publique au Sud; la protection des DPI en tant qu'outil nécessaire à la promotion du commerce et du développement soutenable est indéfectiblement postulée dans les ALE états-uniens.

Considérant le fait que ces ALE négligent grandement d'énumérer ses objectifs et ses principes, il devient pour le moins difficile pour les Partenaires d'interpréter le contenu de ces accords

11 Ces interrogations valent également pour le Nord où l'on regrette trop souvent le développement des "me-too drugs" (Hollis, 2004), où l'on observe inquiet la baisse vertigineuse du nombre de nouvelles entités chimiques mises au point ces dernières décennies (NIHCM Fondation, 2002) et la flambée du prix des médicaments (Public Citizen, 2001). 
dans un sens favorable à la promotion de la santé publique. On est bien loin des contenus de l'AADPIC et de la déclaration ministérielle de Doha qui affirment et soutiennent que les Membres doivent interpréter ces dispositions et la protection des DPI en considérant l'indispensable promotion de la santé publique.

En outre, à l'instar de l'ALE Maroc/Etats-Unis, des accords établissent qu'" une Partie pourra fournir dans sa loi une protection, et une mise en exécution, des droits de propriété intellectuelle plus large que celle requise dans ce chapitre, pourvu que la protection et la mise en exécution additionnelle ne soient pas incompatible avec ce chapitre ". Suivant en cela la position minimaliste adoptée par l'OMC en matière de DPI, les ALE définissent donc des standards minimums et ajoutent que les Parties sont parfaitement libres de prévoir des dispositions plus strictes. Sans surprise, ces standards minimums donnent déjà lieu à des dispositions plus contraignantes que celles requises par l'AADPIC; on parle alors de dispositions "AADPIC plus".

\subsection{Les dispositions incriminées}

Dans les ALE bilatéraux et régionaux, on peut envisager certaines dispositions comme des obstacles à la capacité des PED à recourir pleinement aux flexibilités prévues par l'AADPIC. Ces flexibilités concernent essentiellement les critères de brevetabilité, la protection des données cliniques, les LO ou encore les IP. Ici, de nombreuses références seront faites à l'ALE Amérique centrale/République dominicaine/Etats-Unis et l'ALE Maroc/EtatsUnis, ce dernier étant défini comme le niveau le plus élevé de protection des DPI obtenu à ce jour par les Etats-Unis dans le cadre d'un ALE.

Tout comme l'AADPIC, l'ALE Maroc/Etats-Unis admet le besoin de critères limitant la brevetabilité. "Pour protéger l'ordre public ou la moralité ; y compris pour protéger les êtres humains, les animaux ou les espèces végétales ou la santé ou pour éviter des préjudices sérieux envers l'environnement " (Article 15-9, paragraphe 1), les Partenaires pourront exclure certaines inventions du champ du brevet. Toutefois, si l'AADPIC indique que les Membres "pourront prévenir" la brevetabilité de certains objets (Article 27), l'ALE Maroc/Etats-Unis prescrit que les Partenaires "pourront seulement " exclure du champ de la brevetabilité certaines inventions sur la base des critères donnés ci-dessus. Des circonstances autres que celles prévues plus haut ne pourront pas être évoquées pour prévenir l'octroi d'un brevet pour une invention. Ainsi, les critères de nonbrevetabilité sont limités dans l'ALE Maroc/Etats-Unis et pourraient bien l'être dans d'autres ALE. 
En outre, allant plus loin que les prescriptions de l'AADPIC, les ALE entendent cette fois-ci favoriser une interprétation plus large des critères de brevetabilité. Comme il est prévu dans l'AADPIC et l'ALE Maroc/Etats-Unis, les brevets doivent être accordés pour toute invention, dans tous les champs pour les procédés et les produits. Or, la définition de l'invention est élargie dans l'ALE pour inclure "tout nouvel usage ou toute nouvelle méthode d'utilisation d'un produit connu, y compris les nouveaux usages d'un produit connu pour le traitement des êtres humains et des animaux" (Article 15.9, paragraphe 2). Par conséquent, si une entreprise se voit octroyer un brevet pour le développement d'un médicament, précisément pour une indication thérapeutique, elle pourra obtenir un second brevet pour une nouvelle indication thérapeutique, et ainsi de suite. Cette disposition permet donc aux entreprises d'étendre le champ de la protection associée à un produit en déclarant simplement de nouvelles indications thérapeutiques. Cette possibilité peut donner lieu à des stratégies de prolongement de la durée des brevets, dites " evergreening strategies " dans les PED. Même si le produit n'est pas véritablement nouveau, il pourra recevoir plusieurs brevets pour des innovations incrémentales successives. Cela peut retarder encore et encore le lancement d'un médicament générique et maintenir artificiellement les prix à des niveaux élevés, hors de portée pour les autorités sanitaires, les organisations non gouvernementales (ONG) et les patients au Sud.

Sur ce point, l'ALE Maroc/Etats-Unis établit qu'un nouveau produit est "un produit qui contient une nouvelle entité chimique qui n'a pas encore été approuvée sur le territoire du Partenaire " (Article 15.10, paragraphe 1). Ainsi, si un médicament n'est pas nouveau dans le monde pour la simple raison qu'il a été mis au point et breveté par exemple aux Etats-Unis en 1999, en l'absence de dépôt de brevet au Maroc, ce médicament sera considéré comme nouveau et éligible à la brevetabilité. Il n'y a pas de délai réglementaire qui commande une entreprise ayant breveté un produit dans un pays A de le faire dans un pays B avant un certain temps.

Mises ensembles, ces dispositions offrent des opportunités plus grandes d'obtenir un brevet pour un produit et de retarder d'autant le lancement d'un médicament générique plus abordable dans les PED puisque le jeu de la concurrence est empêché. Plus critiquable, pour les médicaments développés et brevetés avant 1995, et donc non brevetables selon l'AADPIC, une entreprise pourra obtenir un brevet dans un PED au motif : (1) qu'elle n'a pas réclamé de brevet dans ce pays et signifie donc que son médicament est nouveau au regard des dispositions prévues dans l'ALE ratifié ; ou (2) qu'elle met en avant le nouvel usage d'un médicament, toujours selon les dispositions prévues dans l'ALE passé. Au total, la mise en oeuvre de l'AADPIC et la signature d'un ALE dans un PED peuvent 
conjointement générer une complexité et une confusion telles qu'une entreprise pourra véritablement déposer une demande de brevet et consacrer des ressources à défendre son point de vue devant un tribunal en cas de litige. A contrario, les génériqueurs risquent de manquer de ressources et de temps pour tenter d'invalider un brevet avant ou après son octroi.

Ainsi, alors qu'une interprétation plus étroite des critères de brevetabilité peut empêcher la multiplication de stratégies de type " evergreening" et favoriser la commercialisation de médicaments génériques plus abordables dans les PED, l'ALE signé avec le Maroc révèle l'intention des Etats-Unis de promouvoir une interprétation plus large de ces critères pour finalement accroître l'étendue du brevet. Cette intention est parfaitement cohérente avec l'objectif des multinationales du secteur pharmaceutique d'obtenir des brevets successifs pour la même entité chimique, de prolonger leurs monopoles et d'écarter une concurrence générique dans les PED avec un effet ultime négatif sur l'accessibilité.

La durée effective du brevet peut indéniablement être réduite du fait d'exigences réglementaires : le contrôle des données cliniques prend du temps et réduit d'autant l'exploitation effective du brevet. Comme il a été vu précédemment, cela a donné lieu dans les années 80 à un prolongement de la durée du brevet aux Etats-Unis avec la promulgation du Hatch-Waxman Act, mouvement largement suivi dans les autres PD. Pour les PED, l'ALE Amérique centrale/République dominicaine/Etats-Unis prévoit par exemple "qu'un Partenaire devra ajuster le terme du brevet de façon à compenser les délais déraisonnables qui ont lieu lors de l'octroi du brevet ". Le délai déraisonnable est fixé à "plus de cinq ans à partir de la date de dépôt du brevet sur le territoire du Partenaire, ou trois ans après qu'une requête pour examen d'une demande de brevet ait été déposée " (Article 15.9, paragraphe 6a). En conséquence, restaurer le terme du brevet peut repousser sa date d'expiration, retarder l'entrée sur le marché des génériqueurs et reporter la fourniture de médicaments plus abordables. Mais au-delà du brevet, la protection des données cliniques peut également aider les entreprises à construire et prolonger leurs monopoles aux dépens des génériqueurs et des patients.

Quand une entreprise veut lancer un produit, elle doit soumettre des données cliniques qui attestent de la qualité, de la sûreté et de l'efficacité du produit auprès de l'agence du médicament ${ }^{12}$. En cas d'avis favorable, l'entreprise reçoit une autorisation de mise sur le marché (AMM). Partie intégrante du processus de $R \& D$, les données cliniques sont des investissements

${ }^{12}$ La Food and Drug Administration aux Etats-Unis, l'Agence Française de Sécurité Sanitaire des Produits de Santé en France, voire l'Agence Européenne du Médicament pour l'Europe. 
coûteux pour les entreprises: ces données sont le résultat de centaines, voire de milliers d'essais cliniques organisés pour évaluer la qualité, la sûreté et l'efficacité d'un médicament. Pour les génériqueurs, de tels investissements sont inutiles: ils doivent uniquement démontrer la bioéquivalence du médicament par rapport à un princeps et ce faisant se reposer sur les données cliniques déjà soumises par le producteur du princeps ${ }^{13}$. Economisant ainsi des dépenses cliniques importantes, les génériqueurs commercialisent leurs médicaments à des prix plus faibles.

Concernant la divulgation des données cliniques, sous couvert d'ALE, les Etats-Unis travaillent dans deux directions : protéger aussi longtemps que possible les données d'utilisations par des tiers (principalement les génériqueurs) et limiter autant que possible les données qu'une entreprise doit soumettre lors d'une demande d'AMM ou d'un dépôt de brevet. Premièrement, si l'AADPIC commande uniquement la protection des données cliniques "d'un usage commercial injuste" (Article 39.3), les ALE prescrivent laconiquement leur protection pour une durée de cinq ans. Précisément, "si une Partenaire exige, comme condition préalable à l'AMM d'un nouveau produit pharmaceutique ou chimique agricole, la soumission (a) des données de sûreté et d'efficacité ou (b) les preuves d'une autorisation antérieure du produit sur un autre territoire (...), le Partenaire ne permettra pas à des tierces personnes qui n'ont pas le consentement de la personne qui a fourni ces informations de commercialiser un produit sur la base de l'autorisation accordée à la personne soumettant ces information pendant au moins cinq ans pour les produits pharmaceutiques et dix ans pour les produits chimiques agricoles à partir de la date d'autorisation sur le territoire du Partenaire" (Article 15.10, paragraphe 1a).

Ainsi, les génériqueurs désireux de commercialiser la copie d'un médicament dans un pays A ne pourront pas utiliser les données cliniques initialement soumises. Ils devront attendre la fin de la période d'exclusivité couvrant ces données dans le pays A ou procéder à de nouveaux tests cliniques en vue de produire leurs propres données cliniques. Il va sans dire que dans ce dernier cas de figure, des coûts additionnels importants seront supportés et finalement facturés aux patients. Plus discutable, l'éventualité de procéder à de nouveaux tests cliniques pour des médicaments déjà autorisés et utilisés dans d'autres pays pose de graves problèmes éthiques comme le suggère Abbott $(2004)^{14}$. Il reste que la protection

13 Le médicament doit avoir la même activité chimique dans le corps comparé au princeps.

14 De nombreuses ONG manifestent contre le caractère non éthique de cette disposition. Au cours de ces essais cliniques, des patients infectés par le VIH/Sida pourraient par exemple être mis sous placebo alors que la qualité, la sûreté et 
des données cliniques octroie un monopole de fait à l'entreprise qui a initialement soumis les données et retarde d'autant la commercialisation de médicaments génériques plus abordables dans les PED ${ }^{15}$.

Plus encore, dans l'ALE Amérique centrale/République dominicaine/Etats-Unis, une autre disposition prévoit que "si un Partenaire autorise, comme condition d'AMM d'un nouveau produit pharmaceutique ou chimique agricole, des tierces parties à soumettre des éléments concernant la sûreté et l'efficacité d'un produit qui a été antérieurement approuvé dans un autre pays, telle que la preuve d'une AMM antérieure, le Partenaire ne devra pas permettre les tierces parties, sans le consentement de la personne qui a auparavant obtenu une telle autorisation dans l'autre territoire, d'obtenir une autorisation ou de commercialiser un produit sur la base (1) des preuves d'une autorisation antérieure de commercialisation dans un autre territoire ou (2) des informations concernant la sûreté et l'efficacité qui ont été précédemment soumises pour obtenir une AMM dans l'autre territoire pour au moins cinq ans pour les produits pharmaceutiques et dix ans pour les produits chimiques agricoles à partir de la date d'octroi de l'autorisation chez le Partenaire à la personne qui a reçu l'autorisation dans l'autre territoire " (Article 15.10, paragraphe1b). Par ailleurs, l'accord établit qu'"un Partenaire peut exiger que la personne qui a fourni les informations dans l'autre territoire cherche à obtenir une autorisation chez le Partenaire d'ici 5 ans après obtention de l'AMM dans l'autre territoire " (Article 15.10, paragraphe b). Rationnellement, une entreprise aura tendance à adopter la stratégie suivante : elle n'aura pas à déposer une AMM pour assurer l'exclusivité des données dans le pays A puisque celle-ci est assurée pour cinq ans par l'AMM initiale octroyée dans le pays B. Cinq ans plus tard, elle demandera une AMM dans le pays A pour assurer la protection de ses données et prolonger son monopole pour cinq années supplémentaires.

Toutes ces dispositions mises bout à bout permettent d'accorder à toute entreprise une protection des données cliniques et un monopole d'exploitation pour dix ans dans le pays A. Autrement dit, il se passera dix ans avant que les génériqueurs puissent utiliser les données cliniques et commercialiser une copie à un prix plus abordable. Plus problématique, il est des situations où un médicament verra son brevet expirer mais son monopole

l'efficacité des ARV utilisés ont déjà été démontrées dans un PD lors d'essais cliniques antérieurs.

${ }_{15} \mathrm{Au}$ cours du cycle de l'Uruguay, des PD ont réclamé une protection plus forte des données cliniques et ont échoué. Il semble donc qu'à travers les ALE, les Etats-Unis aient réussi à mettre en oeuvre des dispositions plus favorables à la protection des données et ce faisant aux monopoles. 
d'exploitation perdurer dans la mesure où la protection des données court toujours dans le pays A (Abbott, 2004, Rossi, 2006).

Par ailleurs, l'ALE Maroc/Etats-Unis prévoit que de nouvelles données cliniques pourront être protégées pour au moins trois ans à partir de l'AMM obtenue chez le Partenaire (Article 15-10, paragraphe $2 b)$. Cette disposition permettra aux entreprises de prolonger encore et encore la protection des données cliniques, en cas par exemple de nouvel usage d'un produit. Elles obtiendront alors un prolongement de leur monopole grâce à des innovations incrémentales apportées aux produits.

Concernant la divulgation elle-même des informations liées à l'innovation, des efforts sont déployés pour réduire autant que possible les informations révélées. L'ALE Maroc/Etats-Unis prescrit que "chaque Partie devra s'assurer que la divulgation d'une invention revendiquée devra être considérée comme suffisamment claire et complète si elle fournit des informations qui permettent à l'invention d'être fabriquée et utilisée par une personne compétente dans le domaine, sans expérience indue " (Article 15.9, paragraphe 10). A la suite des travaux de Arrow (1962), les DPI ont été justifiés par la nécessité de promouvoir l'innovation et le bien être social. Définie comme un bien public, il y avait un risque que le marché (les entreprises) sous-investissent dans l'innovation et que le bien être social soit menacé. Plus tard, le Hatch-Waxman Act allongeait la durée des brevets contre l'assurance d'une concurrence générique ponctuelle et réelle à l'expiration des brevets ; la condition étant une large divulgation de l'information pour assurer une large diffusion de l'innovation et une accessibilité plus aisée, spécialement parmi les génériqueurs prêts à commercialiser des copies plus abordables dès expiration des brevets. La non divulgation complète et large de l'information constitue un enjeu considérable et un moyen critique entre autres de prévenir la concurrence des génériqueurs en leur assurant un accès limité aux informations concernant les produits. Etant donné les ressources limitées allouées au contrôle des demandes de brevets et d'AMM dans les PED, les autorités réglementaires pourraient faire face à certaines difficultés pour évaluer le caractère clair, complet et suffisant des informations soumises. De fait, elles pourraient avoir davantage tendance à s'en remettre aux brevets et aux AMM déjà accordés dans les PD au lieu de procéder à de nouveaux contrôles des données.

Comme il a été vu plus haut, l'AADPIC prévoit que les PED pourront recourir aux IP pour traiter une urgence nationale ou une pratique anticoncurrentielle. Cette possibilité dépend en pratique du principe d'exhaustivité des droits retenu. L'ALE Maroc/Etats-Unis suggère que des efforts importants seront faits, via les accords bilatéraux et régionaux, pour imposer un principe d'exhaustivité restreignant grandement, voire interdisant tout bonnement le 
recours aux IP. En somme, un principe national ou régional sera dès que possible adopté respectivement dans un accord bilatéral ou régional. A titre d'illustration, L'ALE Maroc/Etats-Unis prévoit que "chaque Partie devra s'assurer que les droits exclusifs du détenteur d'un brevet d'empêcher l'importation d'un produit breveté, ou d'un produit qui résulte d'un procédé breveté, sans le consentement du détenteur $d u$ brevet ne seront pas limités par la vente et la distribution du produit en dehors du pays" (Article 15.9, paragraphe 4). Puisque rien dans l'AADPIC ne régit le principe d'exhaustivité des droits, le Maroc vient d'instaurer un principe national d'exhaustivité et de renoncer à toute possibilité d'importer des médicaments moins chers de l'étranger pour traiter une urgence sanitaire ou couper court à une pratique anticoncurrentielle. Au final, la population pourrait bien souffrir de prix prohibitifs et les autorités supportées des dépenses sociales considérables au moment où elles mettent en place une couverture sociale.

Au sujet de la capacité des PED à délivrer une LO pour les mêmes motifs, la complexité et l'incertitude créées par les dispositions des ALE pourraient sérieusement entamer le recours effectif à cet outil. Dans l'ALE Maroc/Etats-Unis, "le Partenaire mettra en ouvre des mesures dans sa procédure d'AMM pour empêcher, (...), d'autres personnes de commercialiser un produit couvert par un brevet pendant la durée de ce brevet, sauf consentement ou acquiescement du détenteur du brevet " (Article 15.10, paragraphe 4a) ${ }^{16}$. D'un côté, les flexibilités contenues dans l'AADPIC prévoient que les brevets pourront être contournés et une LO délivrée sans le consentement des détenteurs en cas notamment d'urgence nationale. De l'autre, les ALE pourraient simplement empêcher l'AMM d'un médicament protégé par un brevet sans l'autorisation de son détenteur. En d'autres termes, la durée du brevet et la protection des monopoles pourraient l'emporter, même dans des circonstances particulières. En outre, puisque les objectifs et principes des ALE demeurent flous et qu'une référence aux flexibilités manquent essentiellement, une interprétation sensible de ces accords dans le respect de la santé publique s'avère difficile. Ainsi, ces dispositions confuses pourraient générer des discussions et des différends sans fin au sein de l'OMC ou des tribunaux nationaux pour établir quelle disposition adoptée. En conséquence, la fourniture de médicaments génériques plus abordables pourrait bien être retardée.

$\mathrm{Au}$ bout du compte, plusieurs dispositions des ALE pourraient sérieusement compromettre le recours aux flexibilités prévues par l'AADPIC. En tant que moyens utilisés pour accorder des

\footnotetext{
16 Une disposition strictement identique est contenue dans L'ALE Amérique Centrale/République dominicaine/Etats-Unis.
} 
droits de commercialisation exclusifs, le brevet et la protection des données peuvent aider les entreprises à protéger leurs positions de monopole, empêcher le jeu de la concurrence générique et altérer l'accessibilité des médicaments. Il reste que de nombreux accords bilatéraux ou régionaux ont déjà été signés et sont en cours de négociation. Pour autant, on ne doit pas négliger le fait que des dispositions contenues dans l'AADPIC jouent un rôle non négligeable dans ces atteintes faites aux besoins sanitaires des PED.

\section{Concurrence et accès aux traitements dans les pays en voie de développement : quelques faits saillants}

Jusqu'ici il a été question des accords internationaux, des promesses et des menaces qu'ils contiennent pour la santé publique au Sud. Tour à tour, une revue des dispositions qui pourraient empêcher le jeu de la concurrence et l'accès aux traitements dans les PED a été établie. Il sera question à présent des faits saillants, des moyens déployés au Sud pour soutenir l'accès aux traitements avant et après globalisation et harmonisation des régimes de DPI, et des obstacles rencontrés à la lumière des expériences indienne et thaïlandaise.

\subsection{L'impact global d'un régime indien de DPI souple sur le prix des médicaments antisida}

En Inde, la production d'ARV a commencé en 1991. A cette date, Cipla se lance prudemment dans la production des ARV les moins onéreux, ceux dont les contenus en principes actifs sont faibles et les procédés de fabrication sont les moins complexes. Une décennie plus tard, cinq entreprises indiennes, (Cipla, Ranbaxy, Hetero, Aurodindo and Cadila), sont impliquées dans la production d'ARV et plus d'une quinzaine de traitements (mono, bi et trithérapies) est vendue dans le monde (Guennif, 2004).

L'entrée de ces génériqueurs sur le marché des ARV a provoqué une baisse remarquable du prix des traitements (cf. figure ci-dessous). Par exemple, le prix d'une thérapie combinant trois ARV recommandés par l'OMS comme traitement de première intention pour les patients naïfs dans les PED a fortement baissé. Cette baisse a commencé avec l'annonce faite en février 2001 par Cipla de vendre sa trithérapie sous la forme d'un cocktail (le Triomune) à 350 dollars par an et par patient aux $\mathrm{ONG}^{17}$. A cette date, la trithérapie coûte

\footnotetext{
17 Un cocktail ou combinaison à doses fixes est un traitement composé de trois ARV qui se présente sous la forme d'un seul comprimé à prendre deux à trois fois par jour. Ces traitements à doses fixes permettent de réduire le nombre de comprimés à prendre par jour, d'améliorer l'observance chez les patients et de réduire les risques de résistance (OMS, 2006).
} 
931 dollars sous princeps. Dès mars 2001, le prix de la trithérapie sous produits princeps tombe à 727 dollars. De surcroît, la concurrence entre les génériqueurs indiens provoque de nouvelles baisses de prix. Deux mois après l'annonce de Cipla, Hetero se lance dans la bataille et déclare vendre le même cocktail à 347 dollars aux ONG. Quelques mois plus tard, Ranbaxy offre à son tour de fournir le cocktail aux ONG à 295 dollars. Dans un rapport récent, MSF (2006) indique que les prix continuent de baisser. Cipla vendait sa trithérapie à 132 dollars contre 556 dollars sous traitements princeps. Ainsi, la concurrence générique s'est révélée un moyen efficace pour faire chuter le prix des traitements.

\section{Graphique 1. Concurrence générique et prix des thérapies antisida}

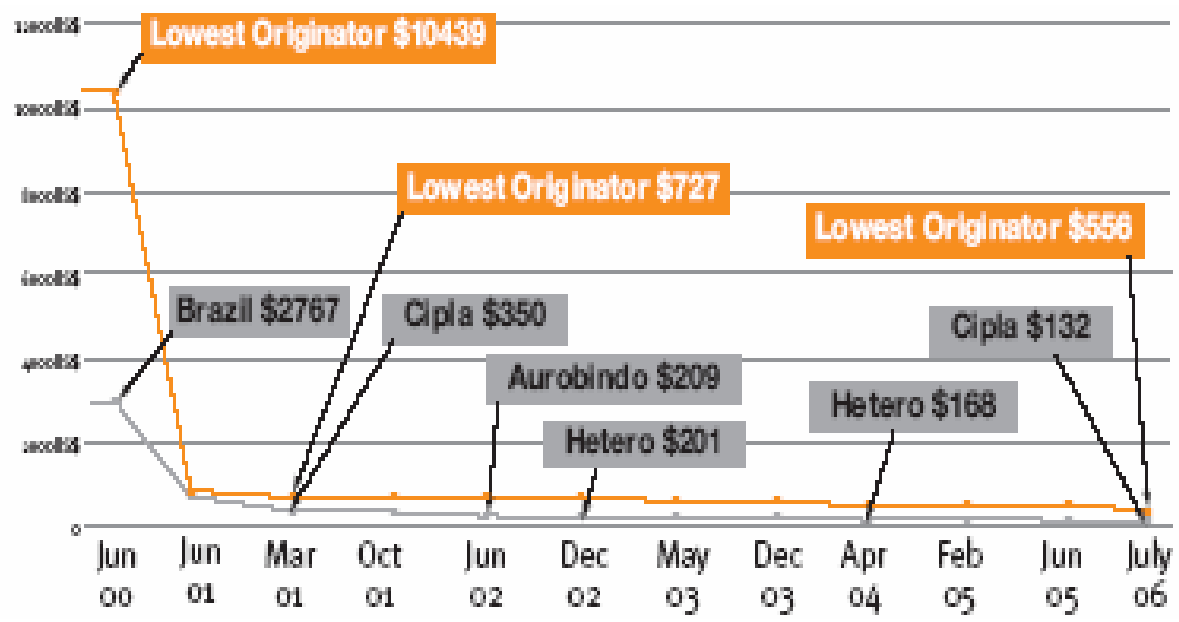

Source: MSF, 2006, Prix les plus bas par patient et par an pour une une tri-thérapie (stavudine, lamivudine et névirapine).

Si l'on s'interroge sur les ressorts de la performance de cette industrie indienne, performance à l'origine de progrès considérables en matière d'accès aux médicaments dans le monde, on trouve une politique du brevet souple adoptée en 1970 (Guennif, 2004, Guennif et Mfuka, 2005). Avant cette date et ce depuis le début du $20^{\text {ième }}$ siècle, les brevets sont octroyés pour les procédés et les produits dans le secteur pharmaceutique. Au lendemain de l'indépendance, on constate que l'industrie indienne est balbutiante et le pays reste fortement dépendant de l'offre extérieure de médicaments commercialisés à des prix prohibitifs (Mittal, 1993, Keayla, 1995, Watal, 2001, Ramana, 2002, Lalitha, 2002). Le système de DPI hérité de la période coloniale est incriminé : il est décrit comme un moyen mis à la disposition des multinationales pour se réserver le marché indien. De fait, elles détiennent entre 80 et $90 \%$ des brevets, 
obtiennent des positions de monopole et pratiquent des prix parmi les plus élevés au monde. En somme, le système de DPI indien a failli à stimuler l'innovation, encourager le développement, améliorer l'autosuffisance sanitaire du pays et promouvoir l'accès aux traitements (Ramana, 2002).

En 1970, 1'Inde amende sa loi sur le brevet en faveur d'un régime plus souple. Désormais, seuls les procédés sont brevetables pour une durée de sept dans le secteur pharmaceutique. Concernant l'usage effectif du brevet ("working patent"), seule la production locale vaut. Contrairement à la période antérieure, l'importation de produits pharmaceutiques ne permet plus de valider l'exploitation effective du brevet. Le détenteur d'un brevet dispose donc d'une période de 3 ans pour exercer ses droits sous la forme d'une production locale. Par ailleurs, l'Inde promeut le contrôle drastique du prix des médicaments en instaurant le Drug Price Control Orders (DPCO) (Singh, 1985, Felker et al., 1997, Srinivasan, 2001, OPPI, 2001, Kunnapallil, 2003) ${ }^{18}$.

En deux décennies, le résultat de ce nouvel "arrangement institutionnel" (Arrow, 1962) est sans équivoque. Une industrie fragmentée comptant des milliers d'unités de production se développe. La production de matière première et celle de formulation augmentent considérablement ${ }^{19}$. Les exportations du secteur sont telles que la balance commerciale affiche un excédent à la fin des années 80. L'Inde fournit en matières premières et/ou en médicaments les grandes multinationales du secteur, les entreprises du Sud (le Brésil par exemple pour son programme anti-sida) et les grands marchés du Nord, le premier étant celui des Etats-Unis grand consommateur de médicaments génériques. L'autosuffisance sanitaire du pays est donc assurée, qui plus est à des prix plus abordables. En somme, la mise en place d'un régime de DPI plus souple, associé à un contrôle drastique des prix, a permis l'essor d'une industrie indienne performante et d'améliorer l'accès aux médicaments dans le pays. Plus tard, cette industrie compétitive a contribué plus largement à faire chuter le prix des ARV et améliorer l'accès aux thérapies dans les PED frappés par l'épidémie de VIH/Sida.

Mais, Membre de l'OMC, l'Inde amende étape par étape (et avec retard) de nouveau sa loi sur le brevet. On peut légitimement se demander en quoi un nouveau renforcement de son régime de DPI

18 Une liste de produits dont le prix est fixé par les pouvoirs publics est éditée régulièrement. Sur cette liste figurent les médicaments les plus essentiels, à savoir ceux dont les volumes de vente sont élevés. Le prix fixé doit permettre aux entreprises de couvrir les coûts des matières premières, de formulation, d'emballage et de distribution, et de recevoir un taux de marge raisonnable.

${ }^{19}$ L'industrie pharmaceutique comprend globalement deux activités principales : (i) la production des matières premières (ou principes actifs), (ii) la formulation ou fabrication des médicaments sur la base de ces matières premières. 
lui permettra de poursuivre ses efforts en matière d'accessibilité aux médicaments (et d'aider l'industrie pharmaceutique à poursuivre sa trajectoire). Pour illustrer les difficultés à attendre de ce nouveau renforcement, on peut souligner le fait que l'OMS a dernièrement changé ses recommandations concernant les traitements de première intention à administrer aux patients naïfs atteints du VIH (OMS, 2006). Désormais, l'Emtricitabine (FTC) et le Tenofovir (TDF) composent les traitements de première intention recommandés. Ces deux ARV ont été brevetés après 1995 et les brevets expireront respectivement en 2015 et 2018. Comme le prévoit l'AADPIC, ces médicaments sont brevetables dans les PED Membres de l'OMC, si par ailleurs la période de transition accordée le permet (2005 ou 2015). En Inde, cette période s'est achevée au 1er janvier 2006 et les entreprises attendent les décisions des contrôleurs pour les demandes de brevet déposées. Dès qu'un brevet sera accordé, les entreprises indiennes n'auront plus la possibilité de produire une copie du médicament protégé et de fournir les autorités publiques, les ONG et les patients en Inde et ailleurs. L'enjeu est prodigieux lorsque l'on sait que les deux ARV précités composent les cocktails recommandés par l'OMS. Ce n'est pas simplement le FTC ou le TDF qui risquent de devenir inabordables en Inde et dans d'autres PED, mais les cocktails composés de l'un ou l'autre de ces ARVs.

Consciente de ces risques pour la santé publique ${ }^{20}$, l'Inde a opté pour une limitation de l'étendue du brevet en restreignent les critères de brevetabilité. Les nouveaux usages ou nouvelles indications thérapeutiques de médicaments déjà connues ne seront pas brevetables (Grace, 2004, Sampath, 2005). De surcroît, un dispositif permettant de faire opposition à une demande de brevet avant son octroi est mis en place. Grâce à ces deux dispositions, la demande de brevet déposée par Novartis pour son Glivec, traitement pour la leucémie, n'a pas été satisfaite. En 2005, les autorités de contrôle ont jugé que le médicament n'était pas nouveau. Actuellement, Novartis conteste cette décision. De même, plusieurs ONG ont fait opposition à la demande de brevet déposée par Gilead pour son TDF. Cette opposition est motivée par le caractère novateur jugé discutable de ce médicament. En contestant la demande de brevet, les ONG entendent prévenir toute entrave à l'accessibilité de ce médicament essentiel. Plus tôt, une opposition a été déposée contre la demande de brevet de GlaxoSmithKline pour le Combivir, un cocktail antisida. Depuis, Gilead a annoncé son intention de céder des licences volontaires aux producteurs indiens pour le TDF dès qu'elle aura obtenu un brevet en Inde (Guennif et Chaisse, 2007).

${ }^{20}$ Et sous la pression d'une partie de l'industrie pharmaceutique indienne. 
En conclusion, n'étant pas engagée dans la signature d'un ALE avec les Etats-Unis pour le moment (néanmoins sujette à des pressions commerciales récurrentes), et consciente de ses obligations en tant que membre de l'OMC, l'Inde tente actuellement d'amender sa loi sur le brevet d'une façon qui lui permette de promouvoir la santé publique. Sur ce point, la présence d'une industrie domestique performante et la préservation d'une concurrence générique vive jouent un rôle considérable. Ainsi, les risques associés aux dispositions "Ouvertes" de l'AADPIC sont pour le moment parfaitement gérés par l'Inde ; gestion qui s'avère plus laborieuse pour la Thaïlande.

\subsection{Le difficile accès aux médicaments sous régime de DPI contraignant en Thaillande}

Dans les années 90, sous pressions commerciales des EtatsUnis, puis suite à la ratification de l'AADPIC, la Thaïlande amende successivement sa loi sur le brevet et met en œuvre un régime de DPI contraignant (Guennif et Mfuka, 2003a, 2003b). Avant 1992, la loi thaïlandaise reconnaît les brevets uniquement sur les procédés et autorise donc les entreprises locales à copier en toute légalité les médicaments brevetés ailleurs. Après cette date, la loi sur la propriété intellectuelle est révisée afin d'introduire les brevets pour les produits. Désormais, tous les médicaments inventés après 1992 sont brevetables en Thaïlande pour une durée de 20 ans durant laquelle aucune version générique ne peut être fabriquée et commercialisée.

Cependant, la non rétroactivité de la loi gêne les multinationales, spécialement états-uniennes qui voient une partie de leurs médicaments copiés par les entreprises locales. Aussi, de nouveau sous pression commerciale, les autorités thaïlandaises mettent en place le Safety Monitoring Programme (SMP). Instauré en 1992, celui-ci a pour but officiellement d'accroître la sécurité et l'efficacité des produits commercialisés localement. En réalité, sous couvert d'objectifs sanitaires, les multinationales obtiennent des droits de commercialisation exclusifs pour les produits qui n'ont jamais fait l'objet d'un dépôt de brevet en Thaïlande. Tant qu'un médicament est placé sous SMP pour s'assurer de sa sûreté et de sa qualité, aucune entreprise locale ne peut produire un générique. Le monopole conféré par le SMP vaut initialement pour 2 ans. Mais, suite à de nouvelles pressions commerciales, cette durée est portée à 
cinq ans. Seulement après expiration de cette période d'exclusivité commerciale, le médicament pourra être copié21.

Par ailleurs, des dispositions sont prévues pour interdire les IP et abolir les LO. Les autorités sanitaires n'ont plus la possibilité d'importer des médicaments vendus à des prix plus bas à l'étranger ou d'autoriser une entreprise locale à produire un générique plus abordable en cas d'urgence nationale ou de pratiques anticoncurrentielles. Au moment où intervient la ratification de l'AADPIC, la Thaïlande a déjà institué un régime de protection des DPI contraignant. En 1999, la loi thaïlandaise est de nouveau amendée pour la rendre compatible avec les dispositions de l'accord multilatéral. A cette occasion, les IP et les LO sont réintroduites dans la législation nationale

Concernant les effets de ces renforcements successifs du régime de DPI, il apparait que les transferts technologiques vers le secteur pharmaceutique restent diffus (Supakankunti et al., 2001, Oxfam, 2001, Guennif et Mfuka, 2005) tandis que les effets préjudiciables à l'accessibilité des médicaments sont parfaitement perceptibles. En effet, entre 1979 et 1992, un générique arrivait sur le marché 1 à 2 ans après la commercialisation du princeps. Après modification du Thai Patent Act en 1992 et introduction du SMP, un générique était disponible 5 ans après le dépôt du brevet, voire 6 ans lorsqu'il s'agit d'un produit mis sous SMP (Kwa, 2001).

De plus, la Thaïlande éprouve les plus grandes difficultés à assurer la fourniture de médicaments à des prix plus abordables, spécialement pour la fourniture d'ARV. Un exemple de ces difficultés concerne "l'épisode ddI". La Government Pharmaceutical Organization (GPO) est une unité de production publique qui a pour fonction de produire les médicaments destinés aux hôpitaux publics. Peu avant la signature de l'AADPIC, cette unité est parvenue à développer une version générique d'un ARV, la ddI, dont le brevet date de 1987 et dont la commercialisation est assurée par BMS. En 1992, date d'entrée en vigueur de la nouvelle loi sur le brevet, BMS demande un brevet pour une formulation améliorée de la ddi ${ }^{22}$. Elle réclame également sa mise sous SMP. Elle obtient ainsi un monopole temporaire. Le médicament devient alors inaccessible puisque GPO n'est plus autorisée à le copier et qu'il est commercialisé à un prix prohibitif par BMS : 2,5 dollars le comprimé lorsque le salaire moyen quotidien avoisine les 3,84 dollars dans le pays (Guennif et Mfuka, 2003a, 2003b).

${ }^{21}$ De fait, en 1998, le SMP concernait près de 700 " nouveaux médicaments " dont certains ARV et favorisait ainsi les détenteurs de brevet au détriment des entreprises nationales de génériques.

${ }^{22}$ Cette amélioration consiste en l'ajout d'un antiacide à la formulation antérieure. 
Loin de renoncer, GPO dépose en 1997 une requête auprès du département thaïlandais de la propriété intellectuelle pour qu'une LO lui soit délivrée. Sous pression des Etats-Unis, le gouvernement thaïlandais renonce et interrompt la procédure de délivrance d'une LO. GPO opte alors pour une autre stratégie: elle propose une nouvelle formulation, de la ddI en poudre pour ne pas violer le brevet détenu par BMS pour sa formulation améliorée. Depuis, les activistes ont porté plainte devant les tribunaux contre BMS en 2001 et réclament le retrait du brevet accordé à la multinationale pour manque d'activité inventive significative, de nouveauté. Le but est d'obtenir que GPO puisse produire la ddI en comprimé, formulation plus commode pour les patients et moins chère. Le brevet n'a pas été invalidé mais son étendue réduite, ce qui permet à GPO de produire un comprimé de 100mg (Oxfam, 2004). Le générique est alors commercialisé à moitié prix comparé au princeps (cf. tableau cidessous). Enfin, sous pression de la société civile, BMS a annoncé dernièrement qu'il renonçait à son brevet

Tableau 1. Comparaison du prix de quelques princeps et génériques en Thaillande (en dollar, en 2001)

\begin{tabular}{llll}
\hline Médicaments & Princeps & Génériques & $\begin{array}{l}\text { Baisses } \\
\text { en } \%\end{array}$ \\
\hline Fluconazole (200mg caps) & 6.20 & 0.26 & 95,8 \\
Stavudine (40mg caps) & 2.60 & 0.10 & 96 \\
Zidovudine (AZT) (100mg caps) & 0,50 & 0.15 & 70 \\
Didanosine (ddI) (100mg tab/170mg en poudre) & 1.20 & 0.62 & 48 \\
\hline
\end{tabular}

Source: Oxfam, 2001, et GPO, 2001.

Un autre exemple des difficultés auxquelles doit faire face la Thaïlande concerne l'annonce faite par les autorités du pays fin 2006 de délivrer des LO pour des médicaments permettant de lutter contre le VIH/Sida et le cancer. Aussitôt, les représentants du commerce des Etats-Unis ont qualifié cette posture de violation des brevets détenus par les multinationales du médicament. Dans un rapport paru quelques mois plus tard, le Ministère de la santé Thaïlandais a dû s'expliquer sur les raisons de sa décision. Tout d'abord, il a été indiqué que la hausse des dépenses de santé est considérable depuis la mise en place d'un programme d'accès universel aux ARV : le budget de ce programme est passé de 10 à 100 millions de dollars entre 2001 et 2007. Ensuite, il a été précisé que la Thaïlande mène depuis deux ans des négociations avec les entreprises détentrices des brevets, Merck et Abbott, pour réduire le prix de plusieurs 
médicaments essentiels, en vain. Enfin, il a été rappelé à toute fin utile que la Thaïlande n'attente nullement aux brevets des multinationales puisque l'AADPIC prévoit la délivrance de LO. Ces précisions n'ont suscité que récriminations et menaces diverses dont celle d'Abbott de ne pas commercialiser certains de ces médicaments dans le pays (Guennif, 2007).

Autre fait important : la Thaïlande est actuellement en pleine négociation avec les Etats-Unis pour la ratification d'un ALE. On peut craindre le contenu et les effets de cet ALE sur la santé publique en considération du régime de DPI restrictif mis en place entre 1992 et 1999 sous pression états-uniennes. Les effets de ce régime sur l'accessibilité des médicaments furent préjudiciables.

\section{Conclusion}

Les éléments présentés ici ont permis d'examiner l'influence potentielle et effective des évolutions récentes des régimes de DPI sur l'accès aux médicaments au Sud. Ces éléments contribuent au débat selon lequel l'instauration d'un système de DPI contraignant conditionnerait le développement socio-économique des PED et l'accès des personnes aux médicaments. Précisément, il est signifié aux PED que l'harmonisation et la globalisation des régimes de DPI inciteront les multinationales à procéder à des transferts technologiques vers le Sud; ce qui aidera au développement socioéconomique des PED en leur permettant d'accéder aux dernières innovations techniques et thérapeutiques.

Pour discuter tout particulièrement du lien supposé soutenable entre régime de DPI contraignant et promotion de la santé publique au Sud, il a été vu que l'AADPIC prévoit certes des flexibilités. Les brevets pourront être contournés en cas notamment d'urgence nationale ou de pratique anticoncurrentielle, et les autorités publiques pourront délivrer des LO ou procéder à des IP. Toutefois, les ALE négociés et signés par les Etats-Unis avec des PED tirent profit des dispositions "ouvertes " de l'AADPIC, omettent d'inclure certaines dispositions précisant les principes et objectifs de ces accords, ou prévoient tout bonnement des dispositions très contraignantes en matière de protection de DPI. Au final, les ALE bilatéraux ou régionaux participent à la mise en place de standards minimums plus élevés que ceux prévus par l'accord multilatéral, en limitant juridiquement les flexibilités prévues par l'AADPIC.

Néanmoins, loin de vouloir simplement procéder à une analyse des dispositions prévues par ces deux types d'accords internationaux et des menaces potentielles qu'ils font peser sur la santé publique, quelques faits stylisés ont permis de fonder quelques craintes. Il a été vu que c'est en assouplissant son régime de DPI que 
l'Inde a amélioré l'accès aux traitements sur la base d'une industrie domestique performante. Que penser donc du nouveau renforcement du régime de DPI que connaît ce pays (et d'autres) suite à la ratification de l'AADPIC lorsque l'on sait par ailleurs que l'industrie indienne a joué ces dernières années un rôle capital dans la chute des prix des ARV dans le monde en développement. Sans présumer de la capacité indienne à poursuivre ses efforts en matière de santé publique, quelques éléments laissent à penser que le pays cherche à tirer profit des flexibilités prévues dans l'AADPIC, en limitant notamment les critères de brevetabilité, pour permettre pour partie à son industrie pharmaceutique de produire et d'exporter. Par ailleurs, poussée par la société civile inquiète, la Thaïlande explore laborieusement la voie des $\mathrm{LO}$ avec la menace récurrente que tous ses efforts en faveur de l'accessibilité des médicaments essentiels soient stoppés et ruinés sous pressions commerciales des Etats-Unis et la signature imminente d'un ALE bilatéral et/ou régional.

Il reste que si le plus sûr moyen d'assurer l'accès des patients aux médicaments était à ce jour l'instauration d'un système de DPI souple, l'enjeu est désormais autre pour les PED. Il est de mettre en place un régime le moins contraignant possible en évitant les surenchères autour des dispositions minimales prévues par l'AADPIC sous pressions commerciales et/ou ratification d'un ALE.

\section{Références bibliographiques}

Abbott F. M. (2006), Intellectual property provisions of bilateral and regional trade agreements in light of US Federal law, Issue Paper $\mathrm{n}^{\circ} 12$, International Centre for Trade and Sustainable Development/United Nations Conference on Trade and Development, Genève.

Abbott F.M. (1996), "Commentary: the international intellectual property order enters the 21 st century", Vanderbilt Journal of Transnational Law, 29, pp. 471-79.

Ainsworth M. (1993), The impact of HIV/AIDS on African development, World Bank, Washington D.C.

Arrow K.J. (1962), "Economic Welfare and the Allocation of Resources for Invention", in R. Nelson (éds.), The Rate and Direction of Inventive Activity, Princeton University Press, pp. 609-25.

Bonnel R. (2000), "HIV/AIDS and economic growth: a global perspective", South African Journal of economics, 68 (5), pp. 820-55.

Boulet P. et G. Velasquez (1999), Mondialisation et accès aux médicaments: les implications des Accords ADPIC/OMC, Organisation Mondiale de la Santé, Genève.

Cohen W., R.R. Nelson et J.P. Walsh (2000), "Protecting their intellectual assets: appropriability conditions and why US 
manufacturing firms patent (or not)", NBER Working Paper Series, $\mathrm{n}^{\circ}$ 7552 .

Correa C. M. (2000), Intellectual property rights, the WTO and developing countries: the TRIPS Agreement and policy options, ZEDTWN, London.

Correa C. M. (2001a), "TRIPS, development and public health", The Journal of World Intellectual Property, 3, pp. 147-62.

Correa C. M. (2001b), Review of the TRIPS Agreement: Fostering the Transfer of Technology to Developing Countries, TWA trade and development Series, $\mathrm{n}^{\circ} 13$.

Correa C. M. (2005), "The TRIPS agreement and transfer of technology", in Gallagher K. P. (éds.), Putting development first, Zed Books, pp. 126-46.

Crampes C. (2000), "La Recherche et la Protection des Innovations dans le secteur pharmaceutique", Revue Internationale de Droit et d'Economie, I (14), pp. 125-46.

Cutler D. et M. MacClellan (2001), "Is technical change in medicine worth it?", Heath Affairs, 20 (5), pp. 11-29.

Demsetz H. (1967), "Toward a Theory of Property Right", American Economic Review, LVII, Mai, pp. 347-67.

Desterbecq H. et B. Remiche (1996), "Les Brevets Pharmaceutiques dans les Accords du GATT: l'Enjeu?", Revue Internationale de Droit Economique, X (1), pp. 7-68.

DiMasi J.A., R.W. Hansen et H.G. Grabowsky (1991), "Cost of innovation in the pharmaceutical industry", Journal of Health Economics, 10, pp. 107-42.

Dixon S., S. MacDonald et J. Roberts (2002), "AIDS and economic growth in Africa: a panel data analysis", Journal of International Development, 13, pp. 381-89.

Drouhin N., V. Touzé et B. Ventelou (2003), "Aids and economic growth in Africa: a critical assessment of the 'base-case scenario' approach", in Moatti J.P., Coriat B., Barnett Y., Souteyrand Y., Dumoulin J. et Flori P.Y. (éds.), Economics of Aids and access to HIV/AIDS care in developing countries. Issues and challenges, ANRS, Collection Sciences Sociales et Sida, pp. 383-413.

Falvey R. et N. Foster (2005), Intellectual property rights, economic growth and technology transfer, United Nations Industrial Development Organization, June, Vienne.

Felker G, S. Chaudhuri, K. György et M. Goldman (1997), The pharmaceutical industry in India and Hungary. Policies, institutions and technological development, World Bank technical paper no. 392, Work in progress for public discussion, World Bank, Genève.

Gallagher K. P. (2005), Putting development first, Zed Books.

Goozner M. (2005), The $\$ 800$ Million Pill: The Truth behind the Cost of New Drugs, University of California Press. 
Grabowsky H. G. (2002), "Patents, innovation and access to new pharmaceuticals", Journal of International Economic Law, 5 (4), pp. 849-60.

Grabowsky H. G. (1982), "Public Policy and Innovation: The Case of Pharmaceuticals", Technovation, 1, pp. 157-89.

Grace C. (2004), The effect of changing intellectual property on pharmaceutical industry prospects in India and China : considerations for access to medicines, Issue Paper, DFID Health systems resource centre, Access to medicines, June.

Guennif S. (2007), "Droits de propriété intellectuelle et santé publique dans les pays du Sud. L'enjeu des médicaments antisida ", Transcontinentales, $n^{\circ} 5$, Second semestre.

Guennif S. (2004), "AIDS in India: Public health related aspects of industrial policy and intellectual property rights in a developing country", CSH Occasional Paper 8, Centre de Sciences Humaines, Publication of the French Research Institutes in India, Rajdhani Art Press, New-Delhi, Inde.

Guennif S. et J. Chaisse (2007), "L'économie politique du brevet au Sud: variations indiennes sur le brevet pharmaceutique ", Revue Internationale de Droit Economique, XXI, 2.

Guennif S. et C. Mfuka (2005), "Promesse et risque du renforcement du brevet au Sud. Transfert technologique, développement pharmaceutique et accès aux traitements", Cahiers de l'Association Tiers Monde, "Droits et développement", n²0, pp. 185-95.

Guennif S. et C. Mfuka (2003a), "Impact of intellectual property rights on AIDS public health policy in Thailand", in Moatti et al. (éds.), Economics of Aids and access to HIV/AIDS care in developing countries. Issues and challenges, ANRS, Collection Sciences Sociales et Sida, pp. 137-51.

Guennif S. et C. Mfuka (2003b), "De la logique de santé publique à la logique industrielle : l'épidémie du sida en Thaïlande", Sciences Sociales et Santé, 21 (1), pp. 75-98.

Hollis A. (2004), "Me-too drugs : is there a problem?", Department of Economics, University of Calgry, Decembre, disponible sur www.who.int/entity/intellectualproperty/topics/ip/MetooDrugs_Hollis 1.pdf.

Keayla B. K. (1995), "Patent protection and the pharmaceutical industry", in Nair K. R. G et A. Kumar (éds.), Intellectual property rights, Allied Publishers Limited, New-Delhi, pp. 151-65.

Kunnapallil P. (2003), "Drudgery of drug price controls: who benefits", Working Paper $n^{\circ} 27$, Center for Civil Society.

Kwa A. (2001), Dying for 'free trade'/dying at the hands of 'free trade' : the law of the jungle institutionalised, Focus on the global South, July. 
Lalitha N. (2002), "Indian pharmaceutical industry in WTO regime: a SWOT analysis", Economic and political weekly, 24, August, pp. 3542-55.

Lall S. (2003), Indicators of the relative importance of IPRs in developing countries, Issue paper $\mathrm{n}^{\circ} 3$, UNCTAD-ICTSD Project on IPRs and sustainable development, June.

Levin R., A. Klevorick, R.R. Nelson et S. Winter (1987), Appropriating the Returns from Industrial Research and Development, Brookings Papers on Economic Activity: Microeconomics.

Lichtenberg F. R. (2001), "Are the benefits of newer drugs worth their costs? Evidence from the 1996 MEPS", Heath Affairs, 20 (5), pp. 24151.

Mansfield E. (1994), Intellectual property Protection, direct investment and technology transfer. Germany, Japon and the United States, Discussion paper 27, International finance corporation.

Mansfield E. (1986), "Patents and innovation: an empirical study", Management science, 32 (2), pp. 173-81.

Maskus K. E. (2004), Encouraging international technology transfer, UNCTAD-ICTSD Project on IPRs and sustainable development, Issue Paper $\mathrm{n}^{\circ} 7$, May.

Maskus K. E. et J.H. Reichman (2005), International public goods and transfer of technology under a globalized intellectual property regime, Cambridge University Press.

Médecins Sans Frontières (2006), Untangling the web of price reductions: a pricing guide for the purchase of ARVs for developing countries, $9^{\text {th }}$ édition, July.

Mfuka C. (2002), "Accord ADPIC et brevets pharmaceutiques - Le difficile accès des pays en développement aux médicaments antisida", Revue d'Economie Industrielle, $\mathrm{n}^{\circ} 99,2^{\text {ème }}$ trimestre, pp. 191-214.

Ministère de la santé publique thaïlandais et Office national de la sécurité sociale (2007), "Facts and evidences on the 10 burning issues related to the government use of patents on three patented essential drugs in Thailand ", Document to support strengthening of social wisdom on the issue of drug patent, White paper, February. Disponible sur www.moph.go.th.

Mittal D. K. (1993), Drugs and pharmaceutical Industry, Anmol publications Pvt. Ltd, New-Delhi.

Musungu S. F. et C. Oh (2006), The use of flexibilities in TRIPS by developing countries: can they promote access to medicines, Commission on Intellectual Property Rights, Innovation and Public health, World Health Organization, April.

National Institute for health Care Management (2002), Changing patterns of pharmaceutical innovation, Research report, May.

Nogues J. (1990), Patents and pharmaceuticals drugs.

Undertanding the pressures on developing countries, WPS 502, 
International Economics Departement, the World Bank, September.

Organisation of Pharmaceutical Producers of India (2001), Drug Prices Control Order - 95, OPPI.

Over M. (1992), The macroeconomic impact of AIDS in sub-Saharan Africa, Technical working paper $\mathrm{n}^{\circ} 3$, World Bank, Washington, D.C.

Oxfam (2004), Free trade agreement between the USA and Thailand threatens access to HIV/AIDS treatment, Oxfam Briefing Note, July.

Public Citizen (2001), Rx R\&D myths: the case against the drug industry's $R \& D$ "scare card", Congress Watch, july. Disponible sur www.citizen.org.

Raizada B. K. et J. Sayed (2002), "IPR and drugs and the pharmaceutical industry-concerns for developing countries", in Dasgupta A. et B. Debroy (éds.), Salvaging the WTO's future: Doha and beyond, New Delhi, Konark, pp. 275-89.

Ramanna A. (2002), "Policy implications of India's patent reforms. Patent applications in the post-1995 era", Economic and political weekly, 25, May, pp. 2065-75.

Rossi F. (2006), "Free trade agreements and TRIPS-plus measures", Int. J. Intellectual Property Management, vol. 1, $\mathrm{n}^{\circ} 1 / 2$.

Saggi K. (2000), Trade, foreign direct investment and international technology transfer. A survey, Policy Research Working Paper, 2349, The World Bank Development Research Group.

Sampath P. G. (2005), Economic aspects of access to medicine after 2005 : product patent protection and emerging firms strategies in the Indian pharmaceutical industry, United Nations University, INTECH, May.

Scherer F. M., 1998, The Patent System and Innovation in Pharmaceuticals, Harvard University Press.

Singh S. (1985), Multinational corporations and Indian Drug Industry, Criterion Publications, New Delhi.

Srinivasan S. (2001), "Drug price control. Who is in charge", Economic and political weekly, 24, March, pp. 997-98.

Supakankunti S., W.S. Janjaroen, O. Tangphao, S. Rahanawijnasini, P. Kraipornsak et P. Pradithavanij (2001), "Impact of the World Trad Organization TRIPS Agreement on the Pharmaceutical Industry in Thailand", Bulletin of the World Health Organization, 79(5), pp. 46170.

UNAIDS (2008), Report on the global AIDS epidemic, UNAIDS.

UNCTAD/ICTSD (2004), Resource book on TRIPS and development: an authoritative and practical guide to the TRIPS agreement, UNCTAD/ICTSD capacity building on IPRs, disponible sur www.iprsonline.org/unctadictsd/ResourceBookIndex.htm.

Velasquez G. et P. Boulet (1999), "Mondialisation et accès aux médicaments. Perspectives sur l'accord ADPIC de l'OMC", Organisation mondiale de la santé, Genève. 
Watal J. (2001), Intellectual property rights in the WTO and developing countries, Oxford University Press.

World Health Organization (2006), Antiretroviral therapy for HIV infection in adults and adolescents in resource-limited settings: towards universal access. Recommendations for a public health approach, revision.

World Trade Organization (2001), "Declaration on the TRIPS agreement and public health", Doha WTO Ministerial 2001, 14th of November 2001, disponible sur www.wto.org.

World Trade Organization (1994), "Agreement on trade-related aspects of intellectual property rights", Annexe 1C, disponible sur www.wto.org. 